\title{
Subwavelength resonant dielectric nanoparticles with high refractive indices
}

H. Ammari and A. Dabrowski and B. Fitzpatrick and P. Millien and

M. Sini

Research Report No. 2019-11

February 2019

Seminar für Angewandte Mathematik

Eidgenössische Technische Hochschule

CH-8092 Zürich

Switzerland 


\title{
Subwavelength resonant dielectric nanoparticles with high refractive indices
}

\author{
Habib Ammari* $\quad$ Alexander Dabrowski* Brian Fitzpatrick $^{\dagger}$ \\ Pierre Millien ${ }^{\ddagger} \quad$ Mourad Sini ${ }^{\S}$
}

\begin{abstract}
This paper aims at understanding the nature of the subwavelength resonant frequencies of dielectric particles with high refractive indices. It is proved that for an arbitrary shaped particle, these subwavelength resonant frequencies can be expressed in terms of the eigenvalues of the Newtonian potential associated with its shape. The enhancement of the scattered field at the resonant frequencies is shown. The hybridization of the subwavelength resonant frequencies of a dimer consisting of high refractive index dielectric nanoparticles is also characterized.
\end{abstract}

Mathematics Subject Classification (MSC2000). 35R30, 35C20.

Keywords. Dielectric nanoparticles, high refractive index, subwavelength resonances, asymptotic expansions.

\section{Introduction}

Nanoscale optics is usually associated with plasmonic resonant structures made of metals such as gold or silver. Plasmonic resonances of nanoparticles can be treated as an eigenvalue problem for the Neumann-Poincaré operator, see $[1,6,7,9]$. However, plasmonic structures suffer from high losses inherent in metals and dissipation due to heating. Recent developments in nanoscale optical physics have led to a new branch of nanophotonics focused on the manipulation of optically induced subwavelength resonances in dielectric nanoparticles with high refractive indices $[14,16,17]$. Resonant high-index dielectric nanostructures form new building blocks which can be used to realize unique functionalities and novel photonic devices [14]. Their study has been established as a new research direction in nanophotonics. Nevertheless, despite strong experimental efforts, mathematical modeling of resonant high-index nanoparticles remains limited. Apart from the case where the particles are disks or spheres, their subwavelength resonant frequencies have not been characterized yet.

In this paper, we consider a dielectric high-index nanoparticle of arbitrary shape and characterize its subwavelength resonances in terms of the eigenvalues of the Newtonian potential

\footnotetext{
*Department of Mathematics, ETH Zürich, Rämistrasse 101, CH-8092 Zürich, Switzerland (habib.ammari@math.ethz.ch, alexander.dabrowski@sam.math.ethz.ch).

${ }^{\dagger}$ ESAT - STADIUS, Stadius Centre for Dynamical Systems, Signal Processing and Data Analytics, Kasteelpark Arenberg 10 - box 2446, 3001 Leuven, Belgium (bfitzpat@esat.kuleuven.be).

${ }^{\ddagger}$ Institut Langevin, 1 Rue Jussieu, 75005 Paris, France (pierre.millien@espci.fr).

${ }^{\S}$ RICAM, Austrian Academy of Sciences, Altenbergerstrasse 69, A-4040, Linz, Austria (mourad.sini@oeaw.ac.at).
} 
associated with its shape. Our formula is closely related to the one established in [15]. Then, we provide an asymptotic formula for the field scattered by a dielectric nanoparticle and estimate the scattering enhancement near its resonant frequencies. We also consider the hybridization phenomenon of a dimer consisting of high refractive index dielectric nanoparticles. We derive asymptotic formulas for the hybridized resonant frequencies, which correspond to monopole and dipole modes.

For simplicity of presentation, we consider the Helmholtz equation as a model for the wave propagation. But one should emphasize that the approach developed here can be extended to the full Maxwell's equations. On the other hand, based on the asymptotic formula for the scattered field derived in this paper, one can characterize the temporal response of resonant dielectric nanoparticles and obtain a time-domain resonant-mode-expansion for the scattered field, which generalizes the time-domain asymptotic formula proved in [4] to the case of a resonant subwavelength particle. This can be easily done by reproducing the calculations presented in [11, Appendix B].

Our results in this paper provide a solid mathematical framework for the analysis of resonant dielectric nanoparticles. They make possible the direct calculation of resonant frequencies and the optimal design of dielectric nanoparticles that resonate at specified frequencies. They can also be applied in the design of dielectric metamaterials and are expected to adavance the applications described in $[12,16,17]$, in particular those concerned with metasurfaces, double-negative all dielectric materials, super-focusing, and wavefront control at the deep subwavelength scale.

\section{Resonant frequencies of dielectric nanoparticles with high refractive indices}

Let $D \Subset \mathbb{R}^{d}$, for $d=2,3$, be a small particle of the form $D=z+\delta B$, where $\delta$ is its characteristic size, $z$ its location, and $B$ is a smooth bounded domain containing the origin. Let $\omega$ denote the frequency, let $\varepsilon \equiv \tau \varepsilon_{c}+\varepsilon_{m}$ inside $D$ and $\varepsilon \equiv \varepsilon_{m}$ outside $D$. Here, $\varepsilon_{c}, \varepsilon_{m}$, and $\tau$ are positive constants. Let $E^{\text {in }}$ be an incident plane wave with frequency $\omega$.

Consider the Helmholtz equation

$$
\left\{\begin{array}{l}
\left(\Delta+\omega^{2} \varepsilon\right) E=0 \text { in } \mathbb{R}^{d}, \\
E-E^{\text {in }} \text { satisfies the Sommerfeld radiation condition. }
\end{array}\right.
$$

From

$$
\left(\Delta+\omega^{2} \varepsilon_{m}\right)\left(E-E^{\text {in }}\right)=-\omega^{2} \tau \varepsilon_{c} E \mathbb{1}_{D} \text { in } \mathbb{R}^{d},
$$

where $\mathbb{1}_{D}$ is the characteristic function of $D$, it follows that the following Lippmann-Schwinger representation formula holds:

$$
\left(E-E^{\mathrm{in}}\right)(x)=-\omega^{2} \tau \varepsilon_{c} \int_{D} E(y) \Gamma_{m}(x-y ; \omega) d y \quad \text { for } x \in \mathbb{R}^{d},
$$

where $\Gamma_{m}$ is the outgoing (i.e., subject to the Sommerfeld radiation condition) fundamental solution of $\Delta+\varepsilon_{m} \omega^{2}$ in free space.

Let $k_{m}=\omega \sqrt{\varepsilon_{m}}$. Let the volume integral operator $K_{D}^{k_{m}}$ be defined by

$$
K_{D}^{k_{m}}: E \in L^{2}(D) \mapsto-\int_{D} E(y) \Gamma_{m}(x-y ; \omega) d y \in L^{2}(D) .
$$

It is well known that, due to the weak singularity of the fundamental solution, $K_{D}^{k_{m}}$ is compact. When the norm of $\tau \omega^{2} \varepsilon_{c} K_{D}^{k_{m}}$ is smaller than $1, I-\tau \omega^{2} \varepsilon_{c} K_{D}^{k_{m}}$ is invertible, so (3) can be rewritten as

$$
E(x)=\left(I-\tau \omega^{2} \varepsilon_{c} K_{D}^{k_{m}}\right)^{-1}\left[E^{\text {in }}\right](x) \text { for all } x \in D,
$$


where $I$ denotes the identity operator.

Assume that the characteristic size $\delta$ of the particle $D$ is much smaller than the wavelength $\left.2 \pi / k_{m}\right)$, and let $\omega \rightarrow 0$. The subwavelength resonance problem is then to find an $\omega \in \mathbb{C}$ close to 0 such that $\left(I-\tau \omega^{2} \varepsilon_{c} K_{D}^{k_{m}}\right)^{-1}$ is singular, or equivalently, such that there exists $L^{2}(D) \ni E \not \equiv 0$ with

$$
E(x)+\omega^{2} \tau \varepsilon_{c} \int_{D} E(y) \Gamma_{m}(x-y ; \omega) d y=0, \quad \text { for } x \in D
$$

see [10]. Such an $\omega$ would be a subwavelength resonance for the high refractive index dielectric particle $D$.

Through a Taylor series expansion of the fundamental solution we obtain the following result.

Lemma 2.1. Let $d=3$. Let $K_{D}^{(0)}$ be the Newtonian potential on D, i.e., the operator defined by

$$
K_{D}^{(0)}[E](x)=-\int_{D} E(y) \Gamma(x-y) d y \quad \text { for } x \in D,
$$

with $\Gamma(x)$ being the fundamental solution of the Laplacian in $\mathbb{R}^{3}$. The operator $K_{D}^{k_{m}}$ can be rewritten as

$$
K_{D}^{k_{m}}=\sum_{i=0}^{\infty} \omega^{i} K_{D}^{(i)}
$$

where the series converges in operator norm if $\omega$ is small enough.

Let $A_{i}=\tau \omega^{2} \varepsilon_{c} K_{D}^{(i)}$. By expanding with a Neumann series, we have

$$
\begin{aligned}
\left(I-A_{0}-\sum_{i=1}^{\infty} \omega^{i} A_{i}\right)^{-1} & =\left(I-\left(I-A_{0}\right)^{-1} \sum_{i=1}^{\infty} \omega^{i} A_{i}\right)^{-1}\left(I-A_{0}\right)^{-1} \\
& =\sum_{k=0}^{\infty}\left(\left(I-A_{0}\right)^{-1} \sum_{i=1}^{\infty} \omega^{i} A_{i}\right)^{k}\left(I-A_{0}\right)^{-1} \\
& =\left(I-A_{0}\right)^{-1}+\left(I-A_{0}\right)^{-1} \omega A_{1}\left(I-A_{0}\right)^{-1}+O\left(\omega^{3}\right) .
\end{aligned}
$$

Recall that $K_{D}^{(0)}: L^{2}(D) \rightarrow L^{2}(D)$ is a compact, self-adjoint operator. Let, for the sake of clarity of the presentation, $\lambda_{0}$ be a simple eigenvalue of $K_{D}^{(0)}$ associated with the normalized eigenfunction $\phi_{0}$ in $L^{2}(D)$. We remark that the eigenvalues of $K_{D}^{(0)}$ are positive. For the analysis of the spectrum of the Newtonian potential, we refer the reader, for instance, to [13].

Let $\omega_{0}$ be a frequency at which $I-A_{0}$ becomes singular. In particular, let

$$
\omega_{0}=1 / \sqrt{\tau \varepsilon_{c} \lambda_{0}} \text {. }
$$

Note that $\omega_{0}$ is small only for $\tau$ large enough. This shows that subwavelength resonances occur only for particles with high refractive indices.

For $\omega$ near $\omega_{0}$, we have, by a pole-pencil operator decomposition, that

$$
\left(I-A_{0}\right)^{-1}[\psi]=\frac{\left\langle\psi, \phi_{0}\right\rangle \phi_{0}}{1-\tau \omega^{2} \varepsilon_{c} \lambda_{0}}+R(\omega)[\psi]
$$

where $\omega \mapsto R(\omega)$ is analytic in a neighborhood of $\omega_{0}$ and $\langle\cdot, \cdot\rangle$ denotes the scalar product on $L^{2}(D)$. Hence, considering only the first two terms in the expansion (5), we obtain from $(2)$ that an approximation of the resonance must satisfy (see, for instance, $[2,5]$ )

$$
\frac{\phi_{0}}{1-\tau \omega^{2} \varepsilon_{c} \lambda_{0}}+\tau \omega^{3} \varepsilon_{c} \frac{\left\langle K_{D}^{(1)}\left[\phi_{0}\right], \phi_{0}\right\rangle}{\left(1-\tau \omega^{2} \varepsilon_{c} \lambda_{0}\right)^{2}} \phi_{0}=0 .
$$

Therefore, we have the following approximation for the subwavelength resonances. 
Proposition 2.2. Let $d=3$ and let $\tau$ be large enough. Let $\omega_{0}$ be defined by (6), where $\lambda_{0}$ is an eigenvalue of the Newtonian potential $K_{D}^{(0)}$. Then, the $O\left(\omega^{4}\right)$-approximation of the subwavelength resonant frequencies $\omega_{\mathrm{s}}$ of the dielectric particle $D$ satisfies

$$
1-\tau \omega_{\mathrm{s}}^{2} \varepsilon_{c} \lambda_{0}=-\tau \omega_{\mathrm{s}}^{s} \varepsilon_{c}\left\langle K_{D}^{(1)}\left[\phi_{0}\right], \phi_{0}\right\rangle .
$$

Note that, in three dimensions,

$$
K_{D}^{(1)}[\phi]=-i \frac{\sqrt{\varepsilon_{m}}}{4 \pi} \int_{D} \phi d y \quad \text { for all } \phi \in L^{2}(D)
$$

Therefore, $\omega_{\mathrm{s}}$ satisfies

$$
1-\tau \omega_{\mathrm{s}}^{2} \varepsilon_{c} \lambda_{0}=\frac{i \tau}{4 \pi} \omega_{\mathrm{s}}^{s} \sqrt{\varepsilon_{m}} \varepsilon_{c}\left(\int_{D} \phi_{0} d y\right)^{2} .
$$

Since $\omega_{\mathrm{s}}$ is close to $\omega_{0}$, by approximating $\omega_{\mathrm{s}}^{3} \simeq \omega_{0}^{3}$, and since by definition $\tau \varepsilon_{c} \lambda_{0}=1 / \omega_{0}^{2}$, we obtain

$$
1-\frac{\omega_{\mathrm{s}}^{2}}{\omega_{0}^{2}}=\frac{i \tau}{4 \pi} \omega_{0}^{3} \sqrt{\varepsilon_{m}} \varepsilon_{c}\left(\int_{D} \phi_{0} d y\right)^{2}
$$

Corollary 2.3. Let $d=3$. Then, the $O\left(\omega^{4}\right)$-approximation of the subwavelength resonant frequencies of the dielectric particle $D$ can be computed as

$$
\omega_{\mathrm{s}}=\omega_{0}-\frac{i}{8 \pi} \frac{\omega_{0}^{2}}{\lambda_{0}} \sqrt{\varepsilon_{m}}\left(\int_{D} \phi_{0} d y\right)^{2}
$$

By using the Lippmann-Schwinger representation formula (3), we can also rewrite

$$
E(x)-E^{\text {in }}(x) \simeq-\omega^{2} \tau \varepsilon_{c} \Gamma_{m}(x-z ; \omega) \frac{\left\langle E^{\text {in }}, \phi_{0}\right\rangle\left(\int_{D} \phi_{0}\right)}{1-\tau \omega^{2} \varepsilon_{c} \lambda_{0}}+\tau \omega^{3} \varepsilon_{c} \frac{\left\langle K_{D}^{(1)}\left[\phi_{0}\right], \phi_{0}\right\rangle\left\langle E^{\text {in }}, \phi_{0}\right\rangle\left(\int_{D} \phi_{0}\right)}{\left(1-\tau \omega^{2} \varepsilon_{c} \lambda_{0}\right)^{2}} .
$$

By plugging the expression of $\omega_{\mathrm{s}}$ obtained in Proposition 2.2 into the above approximation of the scattered field, we arrive at the following result.

Proposition 2.4. For $\omega$ (real) near the resonant frequency $\omega_{\mathrm{s}}$ and $E^{\mathrm{in}}$ such that $\left\langle E^{\mathrm{in}}, \phi_{0}\right\rangle_{L^{2}(D)} \neq$ 0 , the following monopole approximation of the dielectric nanoparticle $D$ holds:

$$
E(x)-E^{\mathrm{in}}(x) \simeq-\frac{\lambda_{0}\left(\frac{\omega_{\mathrm{s}}^{2}}{\omega^{2}}-1\right)-i \frac{\sqrt{\varepsilon_{m}}}{4 \pi}\left(\int_{D} \phi_{0}\right)^{2}\left(\omega-\frac{\omega_{\mathrm{s}}^{3}}{\omega^{2}}\right)}{\left(\lambda_{0}\left(\frac{\omega_{\mathrm{s}}^{2}}{\omega^{2}}-1\right)-i \frac{\sqrt{\varepsilon_{m}}}{4 \pi}\left(\int_{D} \phi_{0}\right)^{2} \frac{\omega_{\mathrm{s}}^{3}}{\omega^{2}}\right)^{2}}\left\langle E^{\mathrm{in}}, \phi_{0}\right\rangle_{L^{2}(D)} \Gamma_{m}(x-z ; \omega),
$$

for $|x-z| \gg 2 \pi /\left(\omega \sqrt{\varepsilon_{m}}\right)$.

Now, we turn to the two-dimensional case. In this case the problem is complicated by the logarithmic singularity of the operator $K_{D}^{k_{m}}$ as $\omega \rightarrow 0$ which gives rise to an averaging operator at leading order when asymptotically expanded. This means we cannot expect to frame the resonance frequency in terms of a single eigenvalue of the Newtonian potential. Instead, in two dimensions the resonance frequency takes account of an infinite number of eigenvalues of the Newtonian potential.

From the asymptotic expansion of the Hankel function $H_{0}^{(1)}$ of the first kind of order zero:

$$
H_{0}^{(1)}(s)=\frac{2 i}{\pi} \sum_{m=0}^{\infty}(-1)^{m} \frac{s^{2 m}}{2^{2 m}(m !)^{2}}\left(\log (\hat{\gamma} s)-\sum_{j=1}^{m} \frac{1}{j}\right),
$$


where $2 \hat{\gamma}=\exp (\gamma-i \pi / 2)$ with $\gamma$ being the Euler's constant (see, for instance, [8]), it follows that

$$
K_{D}^{k_{m}}[E]=-\frac{1}{2 \pi}\left(\log \left(\omega \sqrt{\varepsilon_{m}} \hat{\gamma}\right)\right) \int_{D} E(y) d y+K_{D}^{(0)}+\left(\omega^{2} \log \omega\right) \quad \text { as } \omega \rightarrow 0,
$$

where $K_{D}^{(0)}$ is the Newtonian potential in dimension two, that is, the operator defined on $L^{2}(D)$ by

$$
K_{D}^{(0)}[E](x)=\int_{D} E(y) \Gamma(x-y) d y \quad \text { for } x \in D,
$$

with $\Gamma$ being the fundamental solution of the Laplacian in $\mathbb{R}^{2}$.

Expanding $K_{D}^{k_{m}}$ as in (5) and following the same calculations, we obtain the following characterization of subwavelength resonant frequencies in the two-dimensional case; see Appendix A.

Proposition 2.5. Let $d=2$ and $\tau$ large enough. Then, the $o\left(\omega^{2}\right)$-approximation of the subwavelength resonant frequencies $\omega_{\mathrm{s}}$ of the dielectric particle $D$ satisfies

$$
1-\omega_{\mathrm{s}}^{2} \tau \varepsilon_{c}\left(-\frac{|D|}{2 \pi} \log \left(\omega_{\mathrm{s}} \hat{\gamma} \sqrt{\varepsilon_{m}}\right)+\left\langle K_{D}^{(0)}\left[\hat{\mathbb{1}}_{D}\right], \hat{\mathbb{1}}_{D}\right\rangle\right)=0
$$

where $|D|$ is the volume of $D$ and $\hat{\mathbb{1}}_{D}=\mathbb{1}_{D} / \sqrt{|D|}$.

\section{Hybridization of subwavelength resonant frequencies for a dimer of dielectric nanoparticles}

Consider a dimer of two identical particles $D_{1}$ and $D_{2}$ with the same dielectric parameter as in the above section. Then the field $E-E^{\text {in }}$ scattered by the two particles has the following representation formula:

$$
\left(E-E^{\mathrm{in}}\right)(x)=-\omega^{2} \tau \varepsilon_{c}\left(\int_{D_{1}} E(y) \Gamma_{m}(x-y ; \omega) d y+\int_{D_{2}} E(y) \Gamma_{m}(x-y ; \omega) d y\right) \quad \text { for } x \in \mathbb{R}^{d} .
$$

Define the operators $K_{D_{i}}^{k_{m}}$ and $R_{D_{i}, D_{j}}^{k_{m}}$ for $i, j=1,2$, by

$$
K_{D_{i}}^{k_{m}}:\left.E\right|_{D_{i}} \in L^{2}\left(D_{i}\right) \mapsto-\left.\int_{D_{i}} E(y) \Gamma_{m}(x-y ; \omega) d y\right|_{D_{i}} \in L^{2}\left(D_{i}\right)
$$

and

$$
R_{D_{i}, D_{j}}^{k_{m}}:\left.E\right|_{D_{i}} \in L^{2}\left(D_{i}\right) \mapsto-\left.\int_{D_{i}} E(y) \Gamma_{m}(x-y ; \omega) d y\right|_{D_{j}} \in L^{2}\left(D_{j}\right) .
$$

Then, from (9) we obtain the following system of operator equations:

$$
\left(\begin{array}{cc}
1-\tau \omega^{2} \varepsilon_{c} K_{D_{1}}^{k_{m}} & -\tau \omega^{2} \varepsilon_{c} R_{D_{2}, D_{1}}^{k_{m}} \\
-\tau \omega^{2} \varepsilon_{c} R_{D_{1}, D_{2}}^{k_{m}} & 1-\tau \omega^{2} \varepsilon_{c} K_{D_{2}}^{k_{m}}
\end{array}\right)\left(\begin{array}{l}
\left.E\right|_{D_{1}} \\
\left.E\right|_{D_{2}}
\end{array}\right)=\left(\begin{array}{c}
\left.E^{\mathrm{in}}\right|_{D_{1}} \\
\left.E^{\mathrm{in}}\right|_{D_{2}}
\end{array}\right)
$$

The scattering resonance problem is to find $\omega$ such that the operator in (10) is singular, or equivalently such that there exists $L^{2}\left(D_{1}\right) \times L^{2}\left(D_{2}\right) \ni\left(E_{1}, E_{2}\right) \not \equiv(0,0)$ such that

$$
\left(\begin{array}{cc}
1-\tau \omega^{2} \varepsilon_{c} K_{D_{1}}^{k_{m}} & -\tau \omega^{2} \varepsilon_{c} R_{D_{2}, D_{1}}^{k_{m}} \\
-\tau \omega^{2} \varepsilon_{c} R_{D_{1}, D_{2}}^{k_{m}} & 1-\tau \omega^{2} \varepsilon_{c} K_{D_{2}}^{k_{m}}
\end{array}\right)\left(\begin{array}{l}
\left.E\right|_{D_{1}} \\
\left.E\right|_{D_{2}}
\end{array}\right)=\left(\begin{array}{l}
0 \\
0
\end{array}\right) .
$$

Note that here we have a coupled system of subwavelength resonators. As in $[2,3]$, the following results hold. 
Proposition 3.1. Let $d=3$. The subwavelength resonant frequency $\omega_{\mathrm{s}}$ is hybridized into two subwavelength resonant frequencies $\omega_{\mathrm{s}}^{ \pm}$approximately given by

$$
\omega_{\mathrm{s}}^{ \pm}=\omega_{0} \pm \frac{1}{2} \tau \omega_{0}^{3} \varepsilon_{c} \sqrt{\left(R_{D_{1}, D_{2}}^{\omega_{\mathrm{s}}}\left[\phi_{0}^{(1)}\right], \phi_{0}^{(2)}\right)\left(R_{D_{2}, D_{1}}^{\omega_{\mathrm{s}}}\left[\phi_{0}^{(2)}\right], \phi_{0}^{(1)}\right)}
$$

where $\phi_{0}^{(i)}$, for $i=1,2$, is the eigenfunction associated to the eigenvalue $\lambda_{0}$ of the Newtonian potential of $D_{i}$. Moreover, in the far-field, the dimer of dielectric particles behaves as the sum of a monopole and a dipole.

Now, let $d=2$ and consider for simplicity a dimer of two identical disks $D_{1}$ and $D_{2}$ with the same dielectric parameters as in the above section.

Define the operators $K_{D_{i}}^{k_{m}}$ and $R_{D_{i}, D_{j}}^{k_{m}}$ for $i, j=1,2$, by

$$
\begin{aligned}
K_{D_{i}}^{k_{m}}:\left.E\right|_{D_{i}} \in L^{2}\left(D_{i}\right) & \mapsto-\left.\int_{D_{i}} E(y) \Gamma_{k_{m}}(x-y) d y\right|_{D_{i}} \in L^{2}\left(D_{i}\right), \\
R_{D_{i}, D_{j}}^{k_{m}}:\left.E\right|_{D_{i}} \in L^{2}\left(D_{i}\right) & \mapsto-\left.\int_{D_{i}} E(y) \Gamma_{k_{m}}(x-y) d y\right|_{D_{j}} \in L^{2}\left(D_{j}\right) .
\end{aligned}
$$

Define the operators $M_{D_{i}}^{k_{m}}$ and $N_{D_{i}, D_{j}}^{k_{m}}$ for $i, j=1,2$, by

$$
\begin{aligned}
M_{D_{i}}^{k_{m}} & :=\hat{K}_{D_{i}}^{k_{m}}+K_{D_{i}}^{(0)}, \\
N_{D_{i}, D_{j}}^{k_{m}} & :=\hat{K}_{D_{i}, D_{j}}^{k_{m}}+R_{D_{i}, D_{j}}^{(0)},
\end{aligned}
$$

where

$$
\begin{aligned}
K_{D_{i}}^{(0)}:\left.E\right|_{D_{i}} & \left.\in L^{2}\left(D_{i}\right) \mapsto \int_{D_{i}} E(y) \Gamma(x-y) d y\right|_{D_{i}} \in L^{2}\left(D_{i}\right), \\
\hat{K}_{D_{i}}^{k_{m}}:\left.E\right|_{D_{i}} \in L^{2}\left(D_{i}\right) & \left.\mapsto \log \left(\hat{\gamma} k_{m}\right) \hat{K}_{D_{i}}[E]\right|_{D_{i}} \in L^{2}\left(D_{i}\right), \\
\hat{K}_{D_{i}}:\left.E\right|_{D_{i}} \in L^{2}\left(D_{i}\right) & \mapsto-\left.\frac{1}{2 \pi} \int_{D_{i}} E(y) d y\right|_{D_{i}} \in L^{2}\left(D_{i}\right), \\
R_{D_{i}, D_{j}}^{(0)}:\left.E\right|_{D_{i}} \in L^{2}\left(D_{i}\right) & \left.\mapsto \int_{D_{i}} E(y) \Gamma(x-y)\right|_{D_{j}} \in L^{2}\left(D_{j}\right), \\
\hat{K}_{D_{i}, D_{j}}^{k_{m}}:\left.E\right|_{D_{i}} \in L^{2}\left(D_{i}\right) & \left.\mapsto \log \left(\hat{\gamma} k_{m}\right) \hat{K}_{D_{i}}[E]\right|_{D_{j}} \in L^{2}\left(D_{j}\right), \\
\hat{K}_{D_{i}, D_{j}}:\left.E\right|_{D_{i}} \in L^{2}\left(D_{i}\right) & \mapsto-\left.\frac{1}{2 \pi} \int_{D_{i}} E(y) d y\right|_{D_{j}} \in L^{2}\left(D_{j}\right) .
\end{aligned}
$$

We refer to Appendix C for the proof of the following proposition.

Proposition 3.2. Let $d=2$ and $\tau$ large enough. Then the monopole and dipole hybridized resonances of the dimer of two identical disks $D_{1}$ and $D_{2}$ of radius $\delta$ are approximately given by

$$
1-\omega^{2} \tau \varepsilon_{c}\left(-\frac{\delta^{2}}{2} \log \left(\omega \hat{\gamma} \sqrt{\varepsilon_{m}}\right)(1 \pm 1)+\left\langle K_{D_{1}}^{(0)}\left[\hat{\mathbb{1}}_{D_{1}}\right] \hat{\mathbb{1}}_{D_{1}}\right\rangle \pm\left\langle R_{D_{2}, D_{1}}^{(0)}\left[\hat{\mathbb{1}}_{D_{2}}\right], \hat{\mathbb{1}}_{D_{1}}\right\rangle\right)=0 .
$$

The following corollary gives more explicit formulae for the hybridized resonances in the case when $D_{1}$ and $D_{2}$ are unit disks. 
Corollary 3.3. Let $d=2$ and $\tau$ large enough. Then the monopole and dipole hybridized resonances of a dimer of two identical unit disks $D_{1}$ and $D_{2}$ are given by

$$
\begin{aligned}
& \omega_{\mathrm{m}}(\tau)=-\frac{\sqrt{2} i}{\sqrt{\tau \varepsilon_{c} W(\Phi(\tau))}}, \\
& \omega_{\mathrm{d}}(\tau)=\frac{\sqrt{2}}{\sqrt{\tau \varepsilon_{c}\left(\frac{1}{4}-\frac{2}{\pi}\left\langle R_{D_{2}, D_{1}}^{(0)}\left[\mathbb{1}_{D_{2}}\right], \mathbb{1}_{D_{1}}\right\rangle\right)}},
\end{aligned}
$$

where

$$
\Phi(\tau)=-\frac{2}{\tau \varepsilon_{c}} \exp \left(2 \log \left(\sqrt{\varepsilon_{m}} \hat{\gamma}\right)-\frac{1}{4}-\frac{2}{\pi}\left\langle R_{D_{2}, D_{1}}^{(0)}\left[\mathbb{1}_{D_{2}}\right], \mathbb{1}_{D_{1}}\right\rangle\right),
$$

and $W$ is the lower branch of the Lambert $W$ function defined in the interval $[-1 / e, 0)$.

Remark 3.4. Note that as $\tau \rightarrow \infty$ the monopole resonance $\omega_{\mathrm{m}}=O(1 / \sqrt{\tau \log (\tau)})$ and hence it decays at the same rate as the single particle resonance $\omega_{\mathrm{s}}$, however the dipole resonance $\omega_{\mathrm{d}}$ decays slightly slower, i.e., as $O(1 / \sqrt{\tau})$.

\section{Numerical illustrations}

Let $\varepsilon_{m}=\varepsilon_{c}=1$. Let $D, D_{1}$, and $D_{2}$ be unit disks with $D$ centered at the origin, $D_{1}$ centered at $(-2,0)$, and $D_{2}$ centered at $(2,0)$ with $D$ being the geometry for the single particle problem (3) and $D_{1} \cup D_{2}$ being the geometry for the dimer problem (11).

The asymptotic resonances $\omega_{\mathrm{s}}, \omega_{\mathrm{m}}$, and $\omega_{\mathrm{d}}$ are given by the formulas in Proposition B.1 and Corollary 3.3 , with the $\left\langle R_{D_{2}, D_{1}}^{(0)}\left[\mathbb{1}_{D_{2}}\right], \mathbb{1}_{D_{1}}\right\rangle$ term in the hybridized resonances computed numerically using Python's nquad routine after first putting it in polar coordinates with respect to the center of $D_{1}$. We numerically compute reference solutions to the single particle problem and the dimer problem using boundary integral equation formulations expanded on multipole bases to obtain reference resonances $\omega_{\mathrm{s}, \mathrm{ref}}$ (single particle), $\omega_{\mathrm{m} \text {,ref }}$ (monopole), and $\omega_{\mathrm{d} \text {,ref }}$ (dipole). In Figure 1 we plot the asymptotic resonances along with the corresponding reference resonances and predicted rates of convergence.

In Table 1 we give values of $\omega_{\mathrm{s}}$ and $\omega_{\mathrm{s}, \text { ref }}$ and their corresponding relative errors for $\tau \in$ $\left\{2^{j}\right\}_{j=3}^{7}$.

\begin{tabular}{|r|c|c|c|c|c|}
\hline$\tau$ & $\operatorname{Re}\left(\omega_{\mathrm{s}, \mathrm{ref}}\right)$ & $\operatorname{Re}\left(\omega_{\mathrm{s}}\right)$ & $\operatorname{Im}\left(\omega_{\mathrm{s}, \mathrm{ref}}\right)$ & $\operatorname{Im}\left(\omega_{\mathrm{s}}\right)$ & Rel. err. \\
\hline $2^{3}$ & $2.8012 \mathrm{e}-01$ & $2.8043 \mathrm{e}-01$ & $1.4476 \mathrm{e}-01$ & $1.4022 \mathrm{e}-01$ & $1.44 \mathrm{e}-02$ \\
$2^{4}$ & $1.9685 \mathrm{e}-01$ & $1.9649 \mathrm{e}-01$ & $8.4484 \mathrm{e}-01$ & $8.3198 \mathrm{e}-01$ & $6.23 \mathrm{e}-03$ \\
$2^{5}$ & $1.3602 \mathrm{e}-01$ & $1.3581 \mathrm{e}-01$ & $4.9465 \mathrm{e}-01$ & $4.9189 \mathrm{e}-01$ & $2.40 \mathrm{e}-03$ \\
$2^{6}$ & $9.3188 \mathrm{e}-02$ & $9.3111 \mathrm{e}-02$ & $2.9242 \mathrm{e}-02$ & $2.9221 \mathrm{e}-02$ & $8.22 \mathrm{e}-04$ \\
$2^{7}$ & $6.3606 \mathrm{e}-02$ & $6.3587 \mathrm{e}-02$ & $1.7497 \mathrm{e}-02$ & $1.7521 \mathrm{e}-02$ & $4.68 \mathrm{e}-04$ \\
\hline
\end{tabular}

Table 1: The real and imaginary parts of $\omega_{\mathrm{s}}$ and $\omega_{\mathrm{s}, \mathrm{ref}}$ along with the relative error for $\tau \in$ $\left\{2^{j}\right\}_{j=3}^{7}$. 


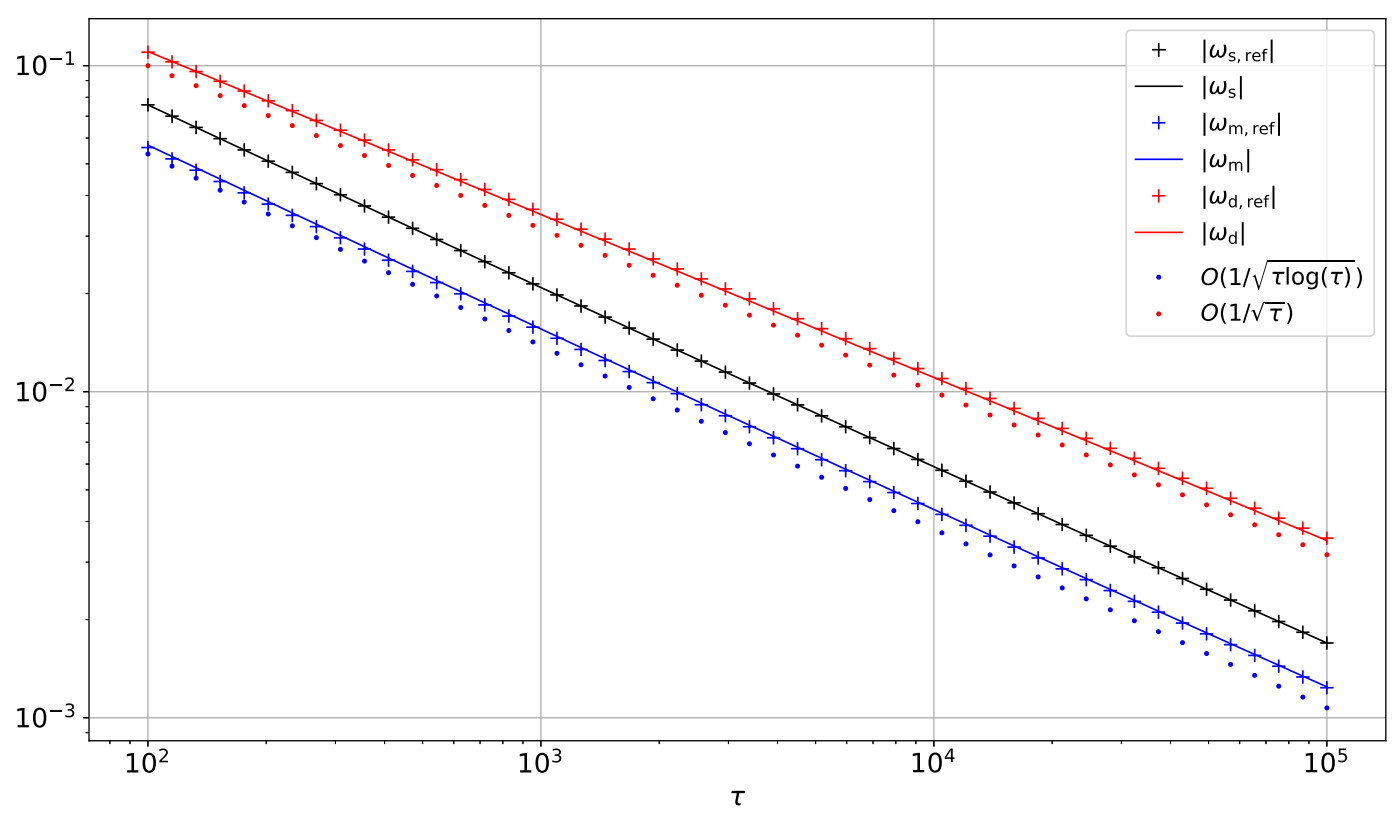

Figure 1: The asymptotic resonances $\omega_{\mathrm{s}}$ (single particle), $\omega_{\mathrm{m}}$ (monpole) and $\omega_{\mathrm{d}}$ (dipole) given by the formulas in Propositions B.1 and Corollary 3.3 and the corresponding resonances obtained using reference solutions.

\section{Concluding remarks}

In this paper, we have provided the first mathematical model of resonant high-index dielectric nanoparticles. We have characterized their subwavelength resonances in terms of the eigenvalues of the associated Newtonian potential. We have also discussed the hybridization phenomenon of a dimer of dielectric nanoparticles with high refractive indices. Our results in this paper pave the way for the analysis, design, and manipulation of resonant dielectric nanostructures and their use as metamaterials. In particular, they can be used for mathematically and numerically modelling super-focusing in dielectric nanostructures, double-negative dielectric materials, and dielectric metasurfaces. Moreover, following [4,11], formula (7) can be easily generalized to the time-domain in order to characterize the temporal response of resonant dielectric nanoparticles and accelerate computations involving the temporal responses of subwavelength dielectric resonators.

\section{A Proof of Proposition 2.5}

Expansion (8) can be rewritten as

$$
K_{D}^{k_{m}}=\hat{K}_{D}^{k_{m}}+K_{D}^{(0)}+O\left(\omega^{2} \log (\omega)\right),
$$

where

$$
\begin{aligned}
\hat{K}_{D}^{k_{m}}[E] & =\log \left(\hat{\gamma} k_{m}\right) \hat{K}_{D}[E] \\
\hat{K}_{D}[E] & =-\frac{1}{2 \pi} \int_{D} E(y) d y \\
K_{D}^{(0)}[E] & =\int_{D} E(y) \Gamma(x-y) d y=-\frac{1}{2 \pi} \int_{D} E(y) \log |\cdot-y| d y .
\end{aligned}
$$


Using this expansion the resonance problem of finding $\omega \in \mathbb{C}$ such that there exists a solution $L^{2}(D) \ni E \not \equiv 0$ to (3) becomes

$$
\left(I-\omega^{2} \tau \varepsilon_{c}\left(\hat{K}_{D}^{k_{m}}+K_{D}^{(0)}\right)\right)[E](x)=O\left(\omega^{4} \log (\omega)\right) .
$$

Denote by

$$
M_{D}^{k_{m}}:=\hat{K}_{D}^{k_{m}}+K_{D}^{(0)},
$$

which is self-adjoint as it is the sum of bounded self-adjoint operators.

Note that when

$$
\omega=\frac{1}{\sqrt{\tau \varepsilon_{c} \lambda_{0}}},
$$

where $\lambda_{0}$ belongs to $\sigma\left(K_{D}^{(0)}\right)$, the spectrum of $K_{D}^{(0)}$, the following equation has a non-trivial solution:

$$
\left(I-\omega^{2} \tau \varepsilon_{c} K_{D}^{(0)}\right)[E]=0 .
$$

Analogously, when

$$
\omega=\frac{1}{\sqrt{\tau \varepsilon_{c} \nu(\omega)}},
$$

where $\nu(\omega) \in \sigma\left(M_{D}^{k_{m}}\right)$ the following equation, which is an approximation of our resonance problem up to order $O\left(\omega^{4} \log (\omega)\right)$, has a non-trivial solution:

$$
\left(I-\omega^{2} \tau \varepsilon_{c} M_{D}^{k_{m}}\right)[E]=0 .
$$

Consider the eigenvalue problem for $M_{D}^{k_{m}}$ :

$$
M_{D}^{k_{m}}[\Psi]=\nu(\omega) \Psi
$$

where $\Psi=\Psi(\omega)$ is normalized on $L^{2}(D)$. Using the expansions

$$
\begin{aligned}
& \Psi(\omega)=\Psi_{0}+O\left(\frac{1}{\log \omega}\right), \\
& \nu(\omega)=\log (\omega) \nu_{0}+\nu_{1}+O\left(\frac{1}{\log \omega}\right),
\end{aligned}
$$

we have

$$
\left(\log (\omega) \hat{K}_{D}+\log \left(\hat{\gamma} \sqrt{\varepsilon_{m}}\right) \hat{K}_{D}+K_{D}^{(0)}\right)\left[\Psi_{0}\right]=\left(\log (\omega) \nu_{0}+\nu_{1}\right)\left[\Psi_{0}\right]+O\left(\frac{1}{\log \omega}\right) .
$$

Equating terms of $O(\log (\omega))$ gives

$$
\hat{K}_{D}\left[\Psi_{0}\right]=\nu_{0} \Psi_{0} .
$$

As $\hat{K}_{D}$ is independent of $x \in D, \Psi_{0}$ must be a constant function, which we normalize on $L^{2}(D)$, i.e., $\Psi_{0}=\hat{\mathbb{1}}_{D}$.

This gives

$$
\nu_{0} \hat{\mathbb{1}}_{D}=\hat{K}_{D}\left[\hat{\mathbb{1}}_{D}\right]=-\frac{|D|}{2 \pi} \hat{\mathbb{1}}_{D},
$$

so $\nu_{0}=-|D| /(2 \pi)$. Next, equating terms of $O(1)$ we have

$$
\begin{aligned}
\nu_{1} \hat{\mathbb{1}}_{D} & =\left(\log \left(\hat{\gamma} \sqrt{\varepsilon_{m}}\right) \hat{K}_{D}+K_{D}^{(0)}\right)\left[\hat{\mathbb{1}}_{D}\right] \\
& =-\frac{|D|}{2 \pi} \log \left(\hat{\gamma} \sqrt{\varepsilon_{m}}\right) \hat{\mathbb{1}}_{D}+K_{D}^{(0)}\left[\hat{\mathbb{1}}_{D}\right],
\end{aligned}
$$


and so, after taking the inner product with $\hat{\mathbb{1}}_{D}$ we get

$$
\nu_{1}=-\frac{|D|}{2 \pi} \log \left(\hat{\gamma} \sqrt{\varepsilon_{m}}\right)+\left\langle K_{D}^{(0)}\left[\hat{\mathbb{1}}_{D}\right], \hat{\mathbb{1}}_{D}\right\rangle
$$

This means that

$$
\nu(\omega)=-\frac{|D|}{2 \pi} \log \left(\hat{\gamma} k_{m}\right)+\left\langle K_{D}^{(0)}\left[\hat{\mathbb{1}}_{D}\right], \hat{\mathbb{1}}_{D}\right\rangle+O\left(\frac{1}{\log \omega}\right) .
$$

Using the expansion of $\nu(\omega)$ in (15) we obtain from (13) that

$$
1-\omega_{\mathrm{s}}^{2} \tau \varepsilon_{c}\left(-\frac{|D|}{2 \pi} \log \left(\omega_{\mathrm{s}} \hat{\gamma} \sqrt{\varepsilon_{m}}\right)+\left\langle K_{D}^{(0)}\left[\hat{\mathbb{1}}_{D}\right], \hat{\mathbb{1}}_{D}\right\rangle\right)=O\left(\frac{\omega^{2}}{\log \omega}\right)=o\left(\omega^{2}\right) .
$$

\section{B The resonance for a unit disk}

Let $D$ be the unit disk. We can obtain a fully explicit expression for (16) in this case as the eigenvalues of the Newtonian potential have a direct relationship with the zeros of the Bessel function of order zero [13]. First we note that $\left\langle K_{D}^{(0)}\left[\hat{\mathbb{1}}_{D}\right], \hat{\mathbb{1}}_{D}\right\rangle=1 / \pi\left\langle K_{D}^{(0)}\left[\mathbb{1}_{D}\right], \mathbb{1}_{D}\right\rangle$. Now, let $J_{l}$ be the Bessel function of order $l$ and define $\mu_{j}^{(0)}$ by

$$
J_{0}\left(\mu_{j}^{(0)}\right)=0, \quad j=1,2, \ldots
$$

According to [13], the eigenvalues of the Newtonian potential for the unit disk are given by

$$
\lambda_{0 j}=\frac{1}{\left(\mu_{j}^{(0)}\right)^{2}}, \quad j=1,2, \ldots,
$$

with the associated orthornormal set of eigenfunctions $\left\{e_{j}\right\}_{j=1}^{\infty}$ given by

$$
e_{j}(r)=\beta_{j} J_{0}\left(\mu_{j}^{(0)} r\right) \text {, }
$$

where

$$
\beta_{j}=\frac{1}{\sqrt{\pi} J_{1}\left(\mu_{j}^{(0)}\right)}
$$

Note that

$$
\left\langle\mathbb{1}_{D}, e_{j}\right\rangle^{2}=\frac{4 \pi}{\left(\mu_{j}^{(0)}\right)^{2}}
$$

Then we have

$$
\begin{aligned}
\left\langle K_{D}^{(0)}\left[\mathbb{1}_{D}\right], \mathbb{1}_{D}\right\rangle & =\sum_{j=1}^{\infty}\left\langle\mathbb{1}_{D}, e_{j}\right\rangle^{2} \lambda_{0 j}=4 \pi \sum_{j=1}^{\infty} \frac{\lambda_{0 j}}{\left(\mu_{j}^{(0)}\right)^{2}} \\
& =4 \pi \sum_{j=1}^{\infty} \frac{1}{\left(\mu_{j}^{(0)}\right)^{4}}=\frac{\pi}{8}
\end{aligned}
$$

where we used the identity $\sum_{j=1}^{\infty} 1 /\left(\mu_{j}^{(0)}\right)^{4}=1 / 32[19]$.

Therefore

$$
\left\langle K_{D}^{(0)}\left[\hat{\mathbb{1}}_{D}\right], \hat{\mathbb{1}}_{D}\right\rangle=\frac{1}{8}
$$


and (16) can be written as

$$
1-\frac{\omega^{2} \tau \varepsilon_{c}}{2}\left(-\log \left(\hat{\gamma} k_{m}\right)+\frac{1}{4}\right)=o\left(\omega^{2}\right) .
$$

The precise dependence of $\omega$ on the contrast parameter $\tau$ can be found by writing the solution to (17) in terms of the Lambert W function [18].

Proposition B.1. The resonance for a unit disk as the contrast $\tau \rightarrow \infty$ is given by

$$
\omega_{\mathrm{s}}(\tau)=-\frac{2 i}{\sqrt{\tau \varepsilon_{c} W(\Phi(\tau))}}+o\left(\frac{1}{\tau \log (\tau)}\right),
$$

where

$$
\Phi(\tau)=-\frac{4}{\tau \varepsilon_{c}} \exp \left(2 \log \left(\sqrt{\varepsilon_{m}} \hat{\gamma}\right)-\frac{1}{2}\right),
$$

and $W$ is the lower branch of the Lambert $W$ function defined in the interval $[-1 / e, 0)$.

Proof. Denote by

$$
\begin{aligned}
& \alpha_{0}(\tau):=\frac{\tau \varepsilon_{c}}{2}, \\
& \alpha_{1}(\tau):=\alpha_{0}\left(\log \left(\sqrt{\varepsilon_{m}} \hat{\gamma}\right)-\frac{1}{4}\right), \\
& \alpha_{2}(\tau):=-\frac{2}{\alpha_{0}} \exp \left(\frac{2 \alpha_{1}}{\alpha_{0}}\right) .
\end{aligned}
$$

Then we can write $(17)$ as

$$
1+\alpha_{0} \omega^{2} \log (\omega)+\alpha_{1} \omega^{2}=o(\omega)^{2}
$$

which leads to

$$
-2 \log (\omega)=\frac{2+2 \alpha_{1} \omega^{2}}{\alpha_{0} \omega^{2}}+o(1)
$$

Then

$$
\frac{1}{\omega^{2}}=\exp \left(\frac{2+2 \alpha_{1} \omega^{2}}{\alpha_{0} \omega^{2}}\right)+o(1)
$$

Next we have

$$
\frac{-2}{\alpha_{0} \omega^{2}} \exp \left(-\frac{2}{\alpha_{0} \omega^{2}}\right)=-\frac{2}{\alpha_{0}} \exp \left(\frac{2 \alpha_{1}}{\alpha_{0}}\right)+o\left(\exp \left(-1 / \omega^{2}\right)\right) .
$$

The Lambert $W$ function is a map $z e^{z} \rightarrow W\left(z e^{z}\right)=z[18]$ and as the expression above is in this form, an application of this map leads to

$$
\begin{aligned}
\frac{-2}{\alpha_{0} \omega^{2}} & =W\left(\frac{2}{\alpha_{0}} \exp \left(-\frac{2 \alpha_{1}}{\alpha_{0}}\right)\right)+o(1) \\
& =W\left(\alpha_{2}\right)+o(1) .
\end{aligned}
$$

Note that the Lambert $\mathrm{W}$ function is double-valued in the interval $[-1 / e, 0)$, which is the interval we need to consider when $\tau \rightarrow \infty$, and we should choose the lower branch, denoted by $W_{-1}$ in the literature, to obtain a physically meaningful resonance.

Now

$$
\omega^{2}=-\frac{2}{\alpha_{0} W\left(\alpha_{2}\right)}+o\left(\omega^{2}\right)
$$


and so

$$
\omega= \pm \frac{i \sqrt{2}}{\sqrt{\alpha_{0} W\left(\alpha_{2}\right)}}+o\left(\omega^{2}\right) .
$$

Noting that $\alpha_{2}=O(1 / \tau)$, as $\tau \rightarrow \infty$ we have the expansion [18]

$$
W\left(\alpha_{2}\right)=\log \left(-\alpha_{2}\right)-\log \left(-\log \left(-\alpha_{2}\right)\right)+\frac{\log \left(-\log \left(-\alpha_{2}\right)\right)}{\log \left(-\alpha_{2}\right)}+\ldots,
$$

which implies that $W\left(\alpha_{2}\right)=O(\log (\tau))$. As $\alpha_{0}=O(\tau)$ this means we have

$$
\omega+o\left(\omega^{2}\right)=O\left(\frac{1}{\sqrt{\tau \log (\tau)}}\right)
$$

and squaring both sides gives

$$
\omega^{2}+o\left(\omega^{3}\right)=O\left(\frac{1}{\tau \log (\tau)}\right)
$$

Thus, upon taking the term with positive real part in (19) we obtain (18).

A coarser approximation which gives a clearer qualitative indication of the dependence of the resonance frequency on the system variables is given in the following corollary.

Corollary B.2. As $\tau \rightarrow \infty$ it holds that

$$
\omega_{\mathrm{s}}(\tau)=\frac{2}{\sqrt{\tau \varepsilon_{c} \log (\tau)}} \frac{1}{\varphi(\tau)}+o\left(\frac{1}{\sqrt{\tau \log (\tau)}}\right),
$$

where

$$
\varphi(\tau)=\left(1-\frac{\log \left(\frac{\varepsilon_{m}}{\varepsilon_{c}} \hat{\gamma}^{2}\right)+\frac{1}{2}}{2 \log (\tau)}\right)
$$

Proof. We have

$$
\begin{aligned}
W\left(\alpha_{2}\right) & =\log \left(-\alpha_{2}\right)+\ldots \\
& =-\log (\tau)+\log \left(\frac{\varepsilon_{m}}{\varepsilon_{c}} \hat{\gamma}^{2}\right)+\frac{1}{2}+\ldots
\end{aligned}
$$

Then

$$
\begin{aligned}
\sqrt{W\left(\alpha_{2}\right)} & =\sqrt{-\log \left(\tau \delta^{2}\right)} \sqrt{\left(1-\frac{\log \left(\frac{\varepsilon_{m}}{\varepsilon_{c}} \hat{\gamma}^{2}\right)+\frac{1}{2}}{\log \left(\tau \delta^{2}\right)}\right)+\ldots} \\
& =i \sqrt{\log (\tau)}\left(1-\frac{\log \left(\frac{\varepsilon_{m}}{\varepsilon_{c}} \hat{\gamma}^{2}\right)+\frac{1}{2}}{2 \log (\tau)}\right)+\ldots
\end{aligned}
$$

Substituting this expression for $\sqrt{W\left(\alpha_{2}\right)}$ into (18) asserts the claim. 


\section{Proof of Proposition 3.2}

We have $\left|D_{1}\right|=\left|D_{2}\right|=\pi \delta^{2}$. Since

$$
\begin{aligned}
K_{D_{i}}^{k_{m}} & =M_{D_{i}}^{k_{m}}+O\left(\omega^{2} \log (\omega)\right), \\
R_{D_{i}, D_{j}}^{k_{m}} & =N_{D_{i}, D_{j}}^{k_{m}}+O\left(\omega^{2} \log (\omega)\right),
\end{aligned}
$$

it holds that

$$
\left(\begin{array}{cc}
I-\tau \omega^{2} \varepsilon_{c} M_{D_{i}}^{k_{m}} & -\tau \omega^{2} \varepsilon_{c} N_{D_{2}, D_{1}}^{k_{m}} \\
-\tau \omega^{2} \varepsilon_{c} N_{D_{1}, D_{2}}^{k_{m}} & I-\tau \omega^{2} \varepsilon_{c} M_{D_{2}}^{k_{m}}
\end{array}\right)\left(\begin{array}{l}
\left.E\right|_{D_{1}} \\
\left.E\right|_{D_{2}}
\end{array}\right)=\left(\begin{array}{c}
O\left(\omega^{4} \log (\omega)\right) \\
O\left(\omega^{4} \log (\omega)\right)
\end{array}\right)
$$

Denote by

$$
\begin{aligned}
\hat{\nu}(\omega) & =-\frac{\delta^{2}}{2} \log \left(\hat{\gamma} k_{m}\right)+\left\langle K_{D_{1}}^{(0)}\left[\hat{\mathbb{1}}_{D_{1}}\right], \hat{\mathbb{1}}_{D_{1}}\right\rangle \\
& =-\frac{\delta^{2}}{2} \log \left(\hat{\gamma} k_{m}\right)+\left\langle K_{D_{2}}^{(0)}\left[\hat{\mathbb{1}}_{D_{2}}\right], \hat{\mathbb{1}}_{D_{2}}\right\rangle, \\
\hat{\eta}(\omega) & =\left\langle N_{D_{1}, D_{2}}^{k_{m}}\left[\hat{\mathbb{1}}_{D_{1}}\right], \hat{\mathbb{1}}_{D_{2}}\right\rangle \\
& =\left\langle N_{D_{2}, D_{1}}^{k_{m}}\left[\hat{\mathbb{1}}_{D_{2}}\right], \hat{\mathbb{1}}_{D_{1}}\right\rangle,
\end{aligned}
$$

with these equalities holding due to the symmetry of the dimer. Furthermore, the symmetry of the dimer also means that

$$
\hat{K}_{D_{i}, D_{j}}^{k_{m}}\left[\hat{\mathbb{1}}_{D_{i}}\right]=\hat{K}_{D_{j}}^{k_{m}}\left[\hat{\mathbb{1}}_{D_{j}}\right]
$$

Denote by $\nu(\omega)$ the eigenvalues of the operators $M_{D_{i}}^{k_{m}}$ such that

$$
\left\langle M_{D_{1}}^{k_{m}}\left[\Psi_{D_{1}}\right], \Psi_{D_{1}}\right\rangle=\nu(\omega)=\left\langle M_{D_{2}}^{k_{m}}\left[\Psi_{D_{2}}\right], \Psi_{D_{2}}\right\rangle,
$$

for eigenfunctions $L^{2}\left(D_{i}\right) \ni \Psi_{D_{i}}(\omega)=\Psi_{D_{i}, 0}+O\left(\frac{1}{\log \omega}\right)=\hat{\mathbb{1}}_{D_{i}}+O\left(\frac{1}{\log \omega}\right)$.

From Section A we know that

$$
\left\langle M_{D_{i}}^{k_{m}}\left[\Psi_{D_{i}}\right], \Psi_{D_{i}}\right\rangle=\nu(\omega)=\hat{\nu}(\omega)+o(1)
$$

Denote by $\eta(\omega)=\left\langle N_{D_{i}, D_{j}}^{k_{m}}\left[\Psi_{D_{i}}\right], \Psi_{D_{j}}\right\rangle$. Note also that

$$
\eta(\omega)=\hat{\eta}(\omega)+o(1)
$$

Therefore, we have the following implicit equation for the hybridized resonances,

$$
1-\omega^{2} \tau \varepsilon_{c}\left(-\frac{\delta^{2}}{2} \log \left(\omega \hat{\gamma} \sqrt{\varepsilon_{m}}\right)(1 \pm 1)+\left\langle K_{D_{1}}^{(0)}\left[\hat{\mathbb{1}}_{D_{1}}\right] \hat{\mathbb{1}}_{D_{1}}\right\rangle \pm\left\langle R_{D_{2}, D_{1}}^{(0)}\left[\hat{\mathbb{1}}_{D_{2}}\right], \hat{\mathbb{1}}_{D_{1}}\right\rangle\right)=o\left(\omega^{2}\right)
$$

\section{References}

[1] H. Ammari, Y. Deng, and P. Millien, Surface plasmon resonance of nanoparticles and applications in imaging, Arch. Ration. Mech. Anal., 220 (2016), 109-153.

[2] H. Ammari, B. Fitzpatrick, H. Kang, M. Ruiz, S. Yu, and H. Zhang, Mathematical and computational methods in photonics and phononics, Mathematical Surveys and Monographs, Vol. 235, American Mathematical Society, Providence, 2018. 
[3] H. Ammari, B. Fitzpatrick, H. Lee, S. Yu, H. Zhang, Double-negative acoustic metamaterials, arXiv:1709.08177.

[4] H. Ammari, P. Garapon, L. Guadarrama Bustos, and H. Kang, Transient anomaly imaging by the acoustic radiation force, J. Differential Equations, 249 (2010), 1579-1595.

[5] H. Ammari, H. Kang, and H. Lee, Layer potential techniques in spectral analysis, Mathematical Surveys and Monographs, 153, American Mathematical Society, Providence, RI, 2009.

[6] H. Ammari and P. Millien, Shape and size dependence of dipolar plasmonic resonance of nanoparticles, J. Math. Pures Appl., DOI: https://doi.org/10.1016/j.matpur.2018.12.001 (arXiv:1804.11092).

[7] H. Ammari, P. Millien, M. Ruiz, and H. Zhang, Mathematical analysis of plasmonic nanoparticles: the scalar case, Arch. Ration. Mech. Anal., 224 (2017), 597-658.

[8] H. Ammari and J.-C. Nédélec, Full low-frequency asymptotics for the reduced wave equation, Appl. Math. Lett., 12 (1999), 127-131.

[9] H. Ammari, M. Ruiz, S. Yu, and H. Zhang, Mathematical analysis of plasmonic resonances for nanoparticles: the full Maxwell equations, J. Differential Equations 261 (2016), 36153669.

[10] H. Ammari and H. Zhang, Super-resolution in high-contrast media, Proc. A. 471 (2015), 20140946, 11 pp.

[11] H. Ammari and H. Zhang, A mathematical theory of super-resolution by using a system of sub-wavelength Helmholtz resonators, Comm. Math. Phys., 337 (2015), no. 1, 379-428.

[12] S. Jahani and Z. Jacob, All-dielectric metamaterials, Nature Nanotechnology, 11 (2016), 23-36.

[13] T.S. Kalmenov and D. Suragan, A boundary condition and spectral problems for the Newton potential, in Modern aspects of the theory of partial differential equations, 187-210, Oper. Theory Adv. Appl., 216, Adv. Partial Differ. Equ. (Basel), Birkhäuser/Springer Basel AG, Basel.

[14] A.I. Kuznetsov, A.E. Miroshnichenko, M.L. Brongersma, Y.S. Kivshar, B. Luk'yanchuk, Optically resonant dielectric nanostructures, Science, 354 (2016), 846-853.

[15] T. Meklachi, S. Moskow, and J.C. Schotland, Asymptotic analysis of resonances of small volume high contrast linear and nonlinear scatterers, J. Math. Phys., 59 (2018), 083502.

[16] Z.-J. Yang, R. Jiang, X. Zhuo, Y.-M. Xie, J. Wang, H.-Q. Lin, Dielectric nanoresonators for light manipulation, Physics Reports, 701 (2017), 1-50.

[17] Q. Zhao, J. Zhou, F. Zhang, and D. Lippens, Dielectric metamaterials, Materialstoday, 12 (2009), 60-69.

[18] R.M. Corless, G.H. Gonnet, D.E. Hare, D.J. Jeffrey, and D.E. Knuth, On the Lambert W function, Advances in Computational mathematics, 5(1) (1996), 329-359.

[19] G.N. Watson, A treatise on the theory of Bessel functions, Second Ed., Cambridge university press, 1995. 